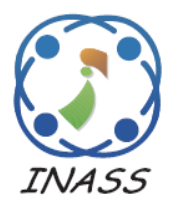

\title{
Robust MIMO-STBC Decoding Technique Using Kurtosis Based Water Cycle Algorithm
}

\author{
Zahraa A. Ghazi ${ }^{1}$ \\ Kasim K. Abdalla ${ }^{1 *}$ \\ ${ }^{I}$ Electrical Engineering Department, Collage of Engineering, Babylon University, Babylon, Iraq \\ * Corresponding author's Email: kasimkaa.11@gmail.com
}

\begin{abstract}
Multiple-input multiple-output (MIMO) systems are used in most modern wireless communication systems, including WiMAX, DVB-NGH, WiFi, HSPA+, LTE, and 4G. MIMO systems with space time coding are a potential technique for increasing data rates and improving wireless communications reliability. This work proposed a new technology for decoding Multiple-Input Multiple-Output Space-Time Block Code (MIMO-STBC) by using blind source separation (BSS), to enhance the BER performance for MIMO STBC channel estimator by using semi-blind independent component analysis (ICA) method, The kurtosis-based source extraction technique is achieved by using real imaginary decomposition $(R-I m)$ of maximum ratio combiner (MRC), Finally, by applying the water cycle method in optimization, we were able to increase the work speed of channel estimation using the ICA technique. The MATLAB software was used to model the system's performance. The simulation results demonstrate that the proposed channel estimation technique is feasible and effective, with only a small difference in performance between the estimated and known channel parameters, such that the proposed algorithm using WCA-kurtosis is better than the performance of the LS algorithm for transmit and receive antennas $2 \times 1,2 \times 2,4 \times 2$, and $4 \times 4$ by $1.6,1.5,1.2$, and 2.2 $\mathrm{dB}$, respectively, at the frame length of the 1024 .
\end{abstract}

Keywords: Space-time block coding (STBC), Water cycle algorithm (WCA), Multiple input multiple output (MIMO), Independent component analysis (ICA), Kurtosis.

\section{Introduction}

In recent years, the need for high-data-rate mobile communication devices has skyrocketed. New approaches are required to meet this massive communications demand while maximizing the efficient use of limited resources such as bandwidth and power. MIMO systems with multiple antenna elements at both link ends are an efficient alternative for future wireless communications systems because they deliver high data rates by utilizing the spatial domain while operating within bandwidth and transmit power limits [1]. STBC is a MIMO transmit method that takes advantage of transmit diversity and excellent reliability orthogonal space time block codes (OSTBCs) and non-orthogonal space time block codes (NOSTCs) are the two types of STBCs (NOSTBCs). The quasi-orthogonal space time block codes (QSTBCs) are a type of NOSTBC that has been the subject of a lot of research. The OSTBCs accomplish complete variety while reducing decoding complexity, albeit at the cost of significant data rate loss. In the case of complex-valued symbol transmission, the full data rate is only possible with full diversity when two transmit antennas (alamouti) are used. With QSTBCs, full data rate may be reached with more than two transmit antennas with just a minor loss of diversity gain $[2,3]$. This made spacetime block codes a very popular and most widely used scheme [4-23].

Many researchers have looked into and studied channel estimation. As a result, several wireless channel estimate algorithms have been proposed and implemented in order to provide channel state information (CSI). WCA has been selected in this work due to, it requires the lower number of insensitive user parameters, where the WCA can address a wide range of optimization problems using the fixed user defined parameters as compared to 
other algorithms, the WCA possesses the advancements of better convergence performance, faster calculation and higher precision compared to the other algorithms. The results confirm that the WCA applied to determine the optimal solution of the populations and can achieve a better result than some other algorithms with an acceptable accuracy and efficiency.

On the receiver side of MIMO communication systems, efficient channel estimation techniques have recently been developed. Estimating methods for MIMO may be classified into three groups [3]:

1- Blind of channel estimation: The receiver in this technique has no prior knowledge of the CSI. This type does not require a training symbol, allowing it to deliver complete throughput. However, the blind estimator's main two flaws are its enormous complexity and latency.

2- Non-blind channel estimation: In this method, the receiver is aware of the CSI. This can be accomplished by utilizing an additional receiving antenna or a longer training sequence period, both of which limit throughput and add to system complexity. 3- Semi-blind of channel estimation: This technique requires fewer of pilots that reduce the efficiency of transmission. The main goal of this paper is to provide STBCs for multiple transmit antennas and multiple receive antennas using semi-blind of channel estimation, improve BER performance, reduce decoding time and solve the ambiguities of BSS.

\section{STBC system MIMO model}

Space time block code is known by $N_{t}$ row and $p$ column transmission matrix $G_{N t}$. The entries of the matrix $G_{N t}$ are linear combination of the variables $S_{l}$, $S_{2}, \ldots, S_{k}$ and their conjugates. different codes were separated using the $\mathrm{N}_{\mathrm{t}}$ of transmitting antennas. Two STBCs $\left(G_{2}, G_{4}\right)$ are utilized in this paper to assess the robustness of the proposed method, and their encoding matrices are as follows:

$$
G_{2}=\left(\begin{array}{rr}
S_{1} & -S_{2}^{*} \\
S_{2} & S_{1}^{*}
\end{array}\right)
$$

where this code known Alamouti STBC, and

$$
G_{4}=\left[\begin{array}{cccc}
S_{1} & S_{2} & S_{3} & S_{4} \\
-S_{2} & S_{1} & -S_{4} & S_{3} \\
-S_{3} & S_{4} & S_{1} & -S_{2} \\
-S_{4} & -S_{3} & S_{2} & S_{1} \\
S_{1}{ }^{*} & S_{2}{ }^{*} & S_{3}{ }^{*} & S_{4}{ }^{*} \\
-S_{2}{ }^{*} & S_{1}{ }^{*} & -S_{4}{ }^{*} & S_{3}{ }^{*} \\
-S_{3}{ }^{*} & S_{4}{ }^{*} & S_{1}{ }^{*} & -S_{2}{ }^{*} \\
-S_{4}{ }^{*} & -S_{3}{ }^{*} & S_{2}{ }^{*} & S_{1}{ }^{*}
\end{array}\right]
$$

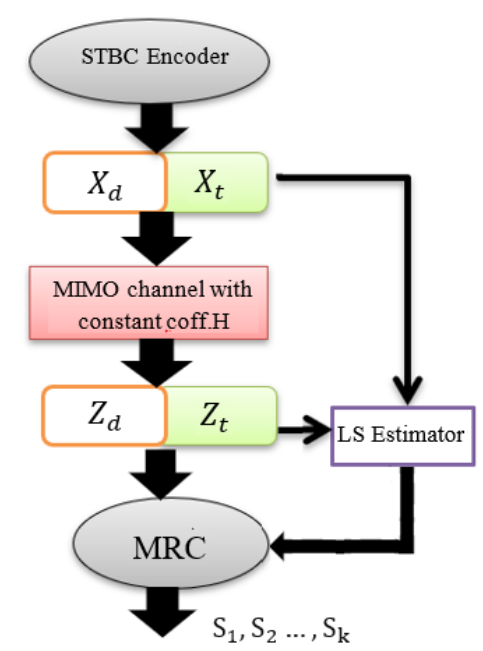

Figure. 1 Channel estimation using pilot sequence

In a MIMO system, matrix notation may be used to express the data transmission process.

Let consider a Flat semi-fixed fade channel with $N_{t}$ transmitters and $N_{r}$ receivers, then received signal vector $\boldsymbol{Z}^{t}=\left[\begin{array}{c}\boldsymbol{Z}_{1}^{t} \\ \vdots \\ \boldsymbol{Z}_{N_{r}}^{t}\end{array}\right]$ at time $t$ is given by $[5,6]$ :

$$
Z^{t}=H X^{t}+\text { noise }
$$

where $X^{t}=\left[\begin{array}{c}x_{1}^{t} \\ \vdots \\ x_{N_{t}}^{t}\end{array}\right]$ is the transmitted signal vector at time $t$ and $H$ is the $N_{r}^{*} N_{t}$ complex number channel coefficients matrix.

In accordance with the flat semi-fixed fade assumption, the channel response varies arbitrarily from one block to the next, but it remains constant throughout the time of a transmission. this time called coherence time [5].

The decoding process is split into two stages. The channel coefficients must first be estimated using a channel estimator. Then, using MRC, the encoded signals $S_{1}, S_{2} \ldots, S_{k}$ will be estimated by using these values. as shown in Fig. 1 [7, 8].

\section{Estimator of the least squares}

The technique of channel estimate is a significant and difficult problem in wireless communication systems. A channel estimate technique provides sufficient information about any distortion delays, interferences, attenuations, interferences, and phase shifts that occur to the signals carried via the channel [9].

As illustrated in Fig. 1 above, the employment of training symbols or (pilot symbols) $\left(X_{t}\right)$ that are known to the receiver side is a typical channel 
estimation method. The training symbols must be set a frequent as the coherence time to maintain track of the time-varying channel characteristics. When training symbols $Z_{t}$ and $X_{t}$ are provided, the least squares (LS) approach is frequently employed for the channel estimation, and estimated channel matrix is given by [10]:

$$
H_{L S}=Z_{t} X_{t}^{H}\left(\mathrm{X}_{\mathrm{t}} X_{t}^{H}\right)^{-1}
$$

However, increasing the amount of pilot symbols $\left(X_{t}\right)$ improves quality of channel estimates while in same time decreasing transmission efficiency.

\section{STBC decoder using maximum ratio combiner (MRC)}

Two STBCs $\left(G_{2}, G_{4}\right)$ are used in this work to test the robustness of the suggested approach, which is given as:

\section{$4.1(2 \times N r)$}

Alamouti STBC $\left(G_{2}\right)$ is given as an example to illustrate MRC decoder works. If a matrix coefficient of $2 \times N_{r}$ MIMO channel is shown as:

$\boldsymbol{H}=\left[\begin{array}{ll}\hbar_{1} & \hbar_{2}\end{array}\right]$

where $\hbar i$ is ith column of $\boldsymbol{H}$.

The received signals can then be classified as: [21]

$$
Z^{1}=\left[\begin{array}{ll}
\hbar_{1} & \hbar_{2}
\end{array}\right]\left[\begin{array}{l}
S_{1} \\
s_{2}
\end{array}\right]+\text { nose, } \text { at } t=1
$$

When the channel coefficients are still constant (a flat semi-fixed fade channel), the received signals can be then classified as [10]:

$$
Z^{2}=\left[\begin{array}{ll}
\hbar_{1} & \hbar_{2}
\end{array}\right]\left[\begin{array}{c}
-s_{2}^{*} \\
s_{1}^{*}
\end{array}\right]+\text { nose, } \text {, at } t=2
$$

Eq. (6) can be rewritten by using the simple mathematical modification as:

$$
\left(\boldsymbol{Z}^{2}\right)^{*}=\left[\begin{array}{ll}
-\left(\hbar_{2}\right)^{*} & \left(\hbar_{1}\right)^{*}
\end{array}\right]\left[\begin{array}{l}
s_{1} \\
s_{2}
\end{array}\right]+\text { nose }
$$

The combining of Eqs. (5) and (7) together to obtain (8) is the main goal of the MRC as:

$$
\left[\begin{array}{l}
Z_{1} \\
Z_{2}
\end{array}\right]=\left[\begin{array}{cc}
\hbar_{1} & \hbar_{2} \\
-\left(\hbar_{2}\right)^{*} & \left(\hbar_{1}\right)^{*}
\end{array}\right]\left[\begin{array}{l}
\boldsymbol{s}_{1} \\
\boldsymbol{s}_{2}
\end{array}\right]+\text { noise }
$$

\section{$4.2(4 \times N r):$}

In the same manner, the MRC for the generator of four transmitter antenna $G_{4}$. Let the channel coefficient matrix be denoted as $H=$ $\left[\begin{array}{llll}\hbar_{1} & \hbar_{2} & \hbar_{3} & \hbar_{4}\end{array}\right]$ where the $i$ th row of $H$ matrix is $\hbar_{i}$ then the received signals are:

- at $t=1$

$$
Z^{1}=\left[\begin{array}{l}
Z_{1}^{1} \\
Z_{2}^{1} \\
Z_{3}^{1} \\
Z_{4}^{1}
\end{array}\right]=\left[\begin{array}{llll}
\hbar_{1} & \hbar_{2} & \hbar_{3} & \hbar_{4}
\end{array}\right]\left[\begin{array}{l}
S_{1} \\
S_{2} \\
S_{3} \\
S_{4}
\end{array}\right]+\text { noise }
$$

- at $t=2$, if channel still constant then:

$$
Z^{2}=\left[\begin{array}{c}
Z_{1}^{2} \\
Z_{2}^{2} \\
Z_{3}^{2} \\
Z_{4}^{1}
\end{array}\right]=\left[\begin{array}{llll}
\hbar_{1} & \hbar_{2} & \hbar_{3} & \hbar_{4}
\end{array}\right]\left[\begin{array}{c}
-S_{2} \\
S_{1} \\
-S_{4} \\
S_{3}
\end{array}\right]+\text { noise }
$$

By using suitable modification Eq. (11) can be rewritten as:

$$
Z^{2}=\left[\begin{array}{llll}
\hbar_{2} & -\hbar_{1} & \hbar_{4} & -\hbar_{3}
\end{array}\right]\left[\begin{array}{l}
S_{1} \\
S_{2} \\
S_{3} \\
S_{4}
\end{array}\right]+\text { noise }
$$

For the same path found the other row of $H_{M R C}$ matrix:

$$
\left[\begin{array}{c}
Z^{1} \\
Z^{2} \\
Z^{3} \\
Z^{4} \\
\left(Z^{5}\right)^{*} \\
\left(Z^{6}\right)^{*} \\
(Z)^{*} \\
\left(Z^{8}\right)^{*}
\end{array}\right]
$$

$\left[\begin{array}{cccc}\hbar_{1} & \hbar_{2} & \hbar_{3} & \hbar_{4} \\ \hbar_{2} & -\hbar_{1} & \hbar_{4} & -\hbar_{3} \\ \hbar_{3} & -\hbar_{4} & -\hbar_{1} & \hbar_{2} \\ \hbar_{4} & \hbar_{3} & -\hbar_{2} & -\hbar_{1} \\ \left(\hbar_{1}\right)^{*} & \left(\hbar_{2}\right)^{*} & \left(\hbar_{3}\right)^{*} & \left(\hbar_{4}\right)^{*} \\ \left(\hbar_{2}\right)^{*} & \left(-\hbar_{1}\right)^{*} & \left(\hbar_{4}\right)^{*} & \left(-\hbar_{3}\right)^{*} \\ \left(\hbar_{3}\right)^{*} & \left(-\hbar_{4}\right)^{*} & \left(-\hbar_{1}\right)^{*} & \left(\hbar_{2}\right)^{*} \\ \left(\hbar_{4}\right)^{*} & \left(\hbar_{3}\right)^{*} & \left(-\hbar_{2}\right)^{*} & \left(-\hbar_{1}\right)^{*}\end{array}\right]\left[\begin{array}{l}S_{1} \\ S_{2} \\ S_{3} \\ S_{4}\end{array}\right]+$ nois




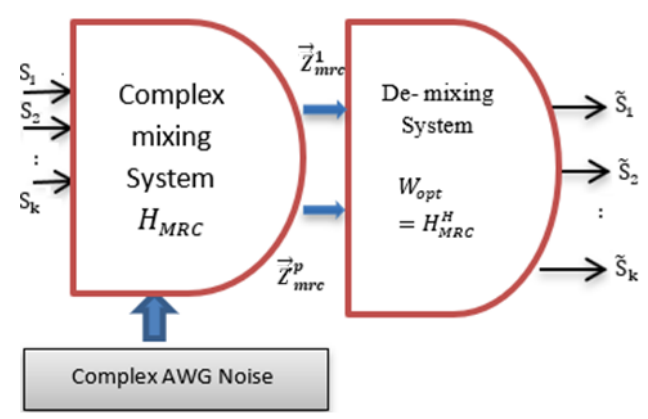

Figure. 2 The MRC as mixing system

In general, the MRC equation for any OSTBC with (m) input samples $S_{1}, S_{2} \ldots, S_{m}$ might be expressed as [12]:

$$
\begin{aligned}
& \mathrm{Z}_{\mathrm{MRC}}=\mathrm{H}_{\mathrm{MRC}} *\left[\begin{array}{c}
\mathrm{S}_{1} \\
\mathrm{~S}_{2} \\
\vdots \\
\mathrm{S}_{\mathrm{m}}
\end{array}\right]+\text { noise } \\
& {\left[\begin{array}{c}
\mathrm{S}_{1} \\
\mathrm{~S}_{2} \\
\vdots \\
\mathrm{S}_{\mathrm{m}}
\end{array}\right]=\frac{1}{\left\|H_{M R C}\right\|}\left(H_{M R C}\right)^{H} \mathrm{Z}_{\mathrm{MRC}}}
\end{aligned}
$$

It can be noticed from previous equations that for any an OSTBC, the matrix of MRC is orthogonal also, which means that $\left(H_{M R C}\right)^{H} H_{M R C}=\left\|H_{M R C}\right\| I_{m}$. The letter I represents the identity matrix, while the letter ()$^{H}$ represents the Hermitian operation. Since $H_{M R C}$ is unitary matrix that made the optimum de mixing matrix is $H_{M R C}^{H}$, the MRC mixing system shown in Fig. 2.

Every statistical way usually deal with complexnumber aren't suitable and give bad performance therefore we will use real-imaginary (R-Im) decomposition for MRC model.

\section{Blind source separation based on kurtosis (BSS)}

Blind signal separation (BSS) is a technique for recovering a single source signal from a noisy mixture of received signals. Kurtosis-based BSE employs a straightforward criterion: the sum of two independent random variables generally has a distribution that is more Gaussian than either of the two original random variables (Central Limit Theorem). As a result, BSS is predicated on the notion that source signals have non-Gaussian distributions. The classical measure of non-Gaussianity is normalized kurtosis where for real random variable $\mathrm{u}$ it could be define as [23].

$$
\operatorname{kurt}(\boldsymbol{u})=\frac{E\left\{\boldsymbol{u}^{4}\right\}}{\left(E\left\{\boldsymbol{u}^{2}\right\}\right)^{2}}-3
$$

Usually, kurtosis-based ICA works for a one-unit ICA (extracting a single source $\boldsymbol{u}), \breve{\boldsymbol{u}}=\boldsymbol{w} \boldsymbol{R}$ Is the solution for one unit ICA transformation, (where $\breve{u}$ represented the estimated of signal source) can be reduced to optimization problem that seeks the best $n_{r}$ dimensional vector $\left(\boldsymbol{w}_{\text {opt }}\right)$ that maximizes the absolute kurtosis measuring. In Other format, the absolute kurtosis is used as the cost function to assess a quality of the mixing vector $(\boldsymbol{w})$ where:

$$
\text { if } \boldsymbol{w}=\left\{\begin{array}{l}
\boldsymbol{w}_{\text {opt }} \rightarrow|\operatorname{kurt}(\boldsymbol{w} \boldsymbol{R})| \approx \max \\
\text { else } \rightarrow|\operatorname{kurt}(\boldsymbol{w R})|<\max
\end{array}\right.
$$

There are two methods for solving optimization problems: the first is the Gradient Ascent-Decent Algorithm (GAA) and evolutionary search algorithm.

\section{GAA for kurtosis based ICA}

The gradient is a 1st order optimization method which tries to discover the function's maximum and minimum values by continually updating the value of $\boldsymbol{w}$ with the cost function's first-order differential. To begin, determine the value of $\frac{\partial \operatorname{kurt}(\breve{u})}{\partial w}$ should evaluate, Then, for each iteration (it), $w$ will be updated recursively using:

$$
\Delta \boldsymbol{w}_{i t}=-\mu \frac{\partial \operatorname{kurt}(\widetilde{\boldsymbol{u}})}{\partial \boldsymbol{w}}
$$

where $\mu$ is the study rate.

The famous update formula is provided by the GAA:

$$
w_{i t+1}=w_{i t}-\mu \varphi(u) R^{T}
$$

where $\boldsymbol{\varphi}(\boldsymbol{u})$ is the nonlinear function and $\mu$ is the learning rate (step size) that can be defined as:

$$
\varphi(\boldsymbol{u})=\frac{\boldsymbol{u}}{E\left\{\boldsymbol{u}^{2}\right\}}-\frac{\boldsymbol{u}^{3}}{E\left\{\boldsymbol{u}^{4}\right\}}
$$

The de-mixing vector will be considered to be $w_{1}$ at the end of each iteration. The other de mixing vectors $w_{2}, w_{3}, \ldots, w_{n_{s}}$ can be easily calculated utilizingstructure of mixing matrix for MIMO-STBC (one source extraction criteria).

The weighted vector must be Normalized for each iteration as follows: $\boldsymbol{w}_{i t+1}=\frac{\boldsymbol{w}_{i t+1}}{\left\|\boldsymbol{w}_{i t+1}\right\|}$, in the end, the algorithm can be stopped if: it exceeds the maximum number of iterations or $\left(\boldsymbol{w}_{\boldsymbol{i} t+1}\right)$ converges to a specified value, for example:

$$
|1-| w_{i t} \times\left(w_{i t+1}\right)^{T}|| \leq \text { Threshold }
$$




\section{Proposed MIMO STBC system mixing model}

To use the BSS method in the decoding of MIMO STBC system, MRC decoder in Eq. (13) will be regarded as the noisy real-value mixing system. and the complex number will then be removed from the equation using real-imaginary (Re-Im) decomposition. Assume the $i_{t h}$ complex source is $\boldsymbol{S}_{\boldsymbol{i}}=\boldsymbol{u}_{\boldsymbol{i}}+\boldsymbol{j} \boldsymbol{u}_{\boldsymbol{k}+\boldsymbol{i}}$ where $u_{i}$ is the real part of $\boldsymbol{S}_{\boldsymbol{i}}, \boldsymbol{u}_{\boldsymbol{k}+\boldsymbol{i}}$ is the imaginary part of $\boldsymbol{S}_{\boldsymbol{i}}$, and $i=1,2, \ldots \ldots, k$. This decomposition will deduce a $n_{s}$ (where $n_{s}=2 k$ ) real value sources arranged as following:

$$
U=\left[\begin{array}{c}
\boldsymbol{u}_{1} \\
: \\
\boldsymbol{u}_{\boldsymbol{k}} \\
\boldsymbol{u}_{\boldsymbol{k}+\mathbf{1}} \\
\vdots \\
\boldsymbol{u}_{\boldsymbol{n}_{s}}
\end{array}\right]=\left[\begin{array}{c}
\operatorname{Re}\left\{\begin{array}{c}
\mathrm{S}_{1} \\
\mathrm{~S}_{2} \\
\vdots \\
\mathrm{S}_{\mathrm{k}}
\end{array}\right\} \\
\operatorname{Im}\left\{\begin{array}{c}
\mathrm{S}_{1} \\
\mathrm{~S}_{2} \\
\vdots \\
\mathrm{S}_{\mathrm{k}}
\end{array}\right\}
\end{array}\right]
$$

Similarly, $Z_{M R C}$ can divied into $n_{r}$ real value combinations in the following manner:

$$
R=\left[\begin{array}{l}
\operatorname{Re}\left\{Z_{M R C}\right\} \\
\operatorname{Im}\left\{Z_{M R C}\right\}
\end{array}\right]=\left[\begin{array}{c}
\boldsymbol{r}_{\mathbf{1}} \\
\boldsymbol{r}_{\mathbf{2}} \\
: \\
\boldsymbol{r}_{\boldsymbol{n}_{\boldsymbol{r}}}
\end{array}\right]
$$

Consider a linear mixing system with $n_{s}$ input sources $U$ and $n_{r}$ output mixes received signals $R$, the $n_{r} \times n_{s}$ mixing matrix $\mathrm{M}$ should look like this:

$$
M=\left[\begin{array}{lr}
\operatorname{Re}\left\{H_{M R C}\right\} & -\operatorname{Im}\left\{H_{M R C}\right\} \\
\operatorname{Im}\left\{H_{M R C}\right\} & \operatorname{Re}\left\{H_{M R C}\right\}
\end{array}\right]
$$

The whole noisy real values mixing system looks like the following equation:

$$
R=M U+\text { noise }
$$

The fact that the mixing matrix is orthogonal

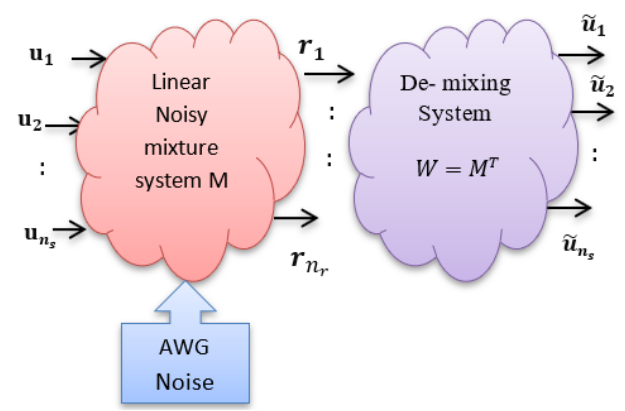

Figure. 3 R-Im decomposition model for MIMO STBC means that the optimum un-normalized de mixing matrix $w_{\text {opt }}$ should equal $M^{T}$, as illustrated in Fig. $4 \mathrm{~b}$.

\section{Statistical analysis of proposed model}

A mathematical representation of a QPSK signal with a carrier amplitude of $A_{C}$ is $S_{i}=\frac{A_{C}}{\sqrt{2}}\{(\mp 1)+$ $j(\mp 1)\}$. After Source Decomposition all sources (normalized sources) are belong to two values $u_{i} \in$ $\{-1,+1\}$. To put it another way, any source may be represented statistically as a discrete r.v. with two level binomial distributions as illustrated in Fig. 4.

Note that $P_{U}(u=-1)=P_{U}(u=+1)=0.5$ is assumed because in a communication system, any source encoding produces a binary signal (modulator input) with an equal likely distribution. The expected statistic for $U$, according to traditional statistical computation analysis:

All sources are sub-Gaussian.

1- The variance is 0.5 and the mean value is 0 .

2- For each source, the normalized Kurtosis is 2

3- The entropy for each source is $\mathbf{1}$.

4- Uncorrelated the All source.

Receiving signals $R$ can be represented as a continuous random variable (r. v.) with a Gaussian distribution probability density function, according to central limit theory. The expected statistic for $\boldsymbol{R}$ are:

1) Because all sources and noise are zero, the mean value of $\mathrm{R}$ is zero.

2) Variance of $\boldsymbol{R}$ depending on noise variance (SNR value).

3) Entropy of $\boldsymbol{R}$ is greater than entropy of $\boldsymbol{U}$.

4) All mixtures received signals are correlated signals.

The predicted signal can be approximated as $\widetilde{\boldsymbol{u}}=$ $\boldsymbol{\alpha u}+$ noise, because the de-mixing method isolates

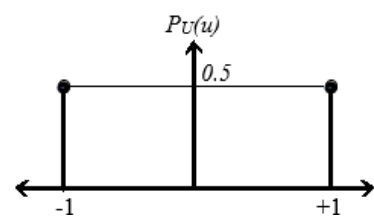

(a)

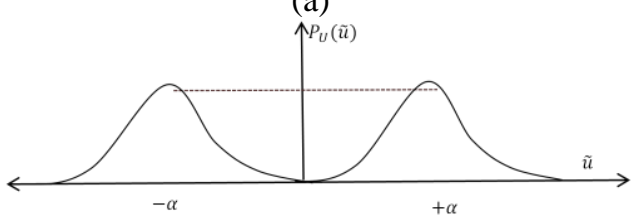

(b)

Figure. 4 (a) Probability mass function of a discrete r.v.u and (b) is the probability density function of $\tilde{u}$. 
the mixture signals without reducing additive noise. The predicted PDF for estimated signal $\tilde{u}$ is based on the additive probity of the two variables is:

$$
P_{U}(\tilde{u})=P_{U}(u) \circledast P_{\text {noise }}
$$

Where the convolution operation is $\circledast$ and $P_{\text {noise }}$ is PDF of noise (zero mean Gaussian noise), the expected PDF of $\tilde{u}$ can be represent as shown in Fig. 4 (b).

\section{Improve kurtosis based ICA using water cycle algorithm (WCA)}

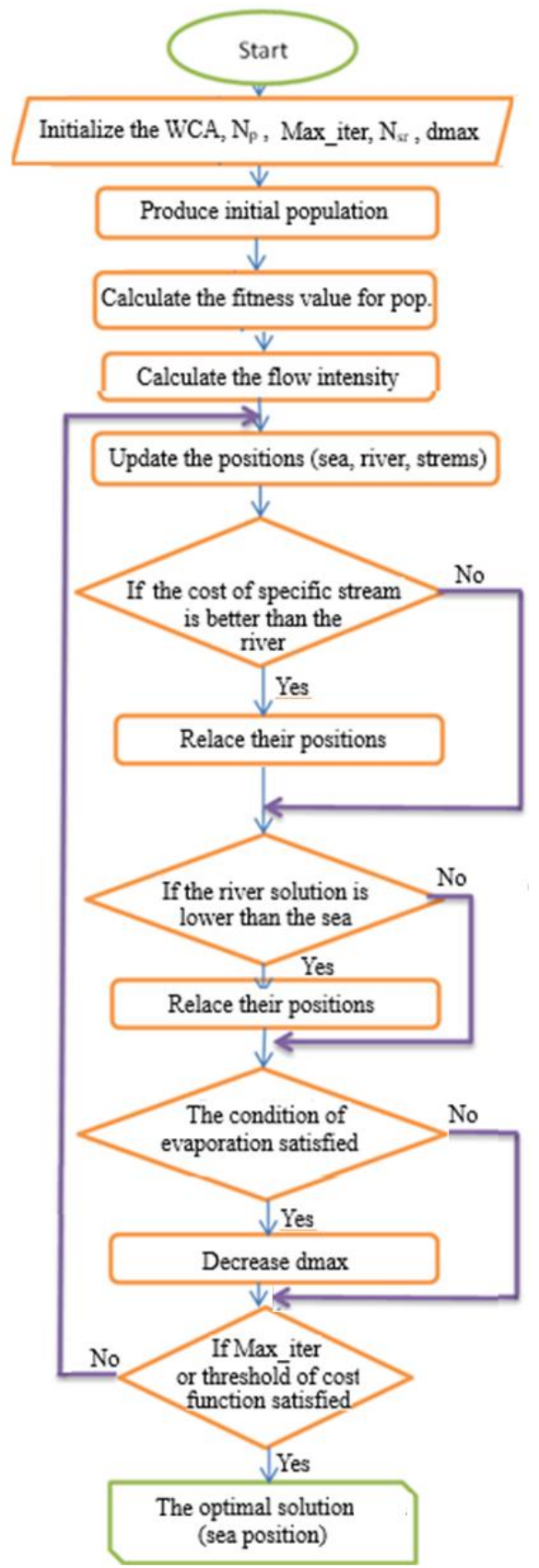

Figure. 5 Water cycle algorithm
In this section we try to combine one unit kurtosis ICA optimization problems, with a high dimensional search-space, using WCA algorithms of optimization. Most of optimization algorithms mimic physical or biological processes. Some of most famous of these algorithms are ant colony optimization [ACO], genetic-algorithm [GA], particle swarm optimization [PSO] and bacterial foraging algorithm [BFA]. There is, however, no specific algorithm for achieving the optimum answer to all optimization issues. Some algorithms provide a better answer than others in certain situations. In this paper the water cycle algorithm was used to improve the efficiency of the LS algorithm, where kurtosis was used as an objective function to reach to the optimal solution.

The WCA is formed by watching a water cycle process and simulates the flow of rivers and streams toward the sea. Assume that rain or precipitation has occurred. Following raining process, a random population (pop) of the design variables (i.e., streams population) is produced. The better individual (i.e., best stream) is picked based on having the smallest cost function (for the minimization issues), is chosen as the sea [22]. Then, the number of good streams (i.e., the cost function values near to the current best record) is chose as rivers, while the other streams flow into the rivers and the sea. WCA can be simplified in Fig. 5.

Starting the optimization method necessitates the formation of an initial population, which is representing by a matrix of streams of size $\mathrm{N}_{\text {pop }} \times \mathrm{N}$, hence, this matrix, which is generated randomly, is given as:

$$
\begin{gathered}
\text { total pop. }=\left[\begin{array}{c}
\text { sea } \\
\text { River }_{1} \\
\text { River }_{2} \\
\vdots \\
\text { Stream }_{1} \\
\text { Stream }_{2} \\
\vdots \\
\\
\text { Stream }_{N_{P O P}}
\end{array}\right] \\
=\left[\begin{array}{cccc}
x_{1}^{1} & x_{2}^{1} & & x_{N}^{1} \\
x_{1}^{2} & x_{2}^{2} & \cdots & x_{N}^{2} \\
& \vdots & \ddots & \vdots \\
x_{1}^{N_{P O P}} & x_{2}^{N_{P O P}} & \cdots & x_{N}^{N_{P O P}}
\end{array}\right]
\end{gathered}
$$

where $N_{p o p}$ and $N$ are the total population number and the number of design variables, respectively. For continuous and discrete issues, each of the decision variable values $\left(x_{1}, x_{2}, \ldots, x_{N}\right)$ can be expressed as a floating-point number (real values) or as a predetermined set. The cost of a stream is determined 
by evaluating the cost function $(C)$, which is shown below:

$$
C_{i}=\operatorname{Cost}_{i}=f\left(x_{1}^{i}, x_{2}^{i}, \ldots, x_{N}^{i}\right), i=1,2, . ., N_{\text {pop }}
$$

The cost function is absolute kurtosis (that given in Eq. (15)) for each individual.

The initial sea position should equal to the first column of $M_{L S}$, then the other population (the position of streams and rivers) are generated randomly around the initial position of sea where distance doesn't exceed \pm 1 initial value This distance is terrifying

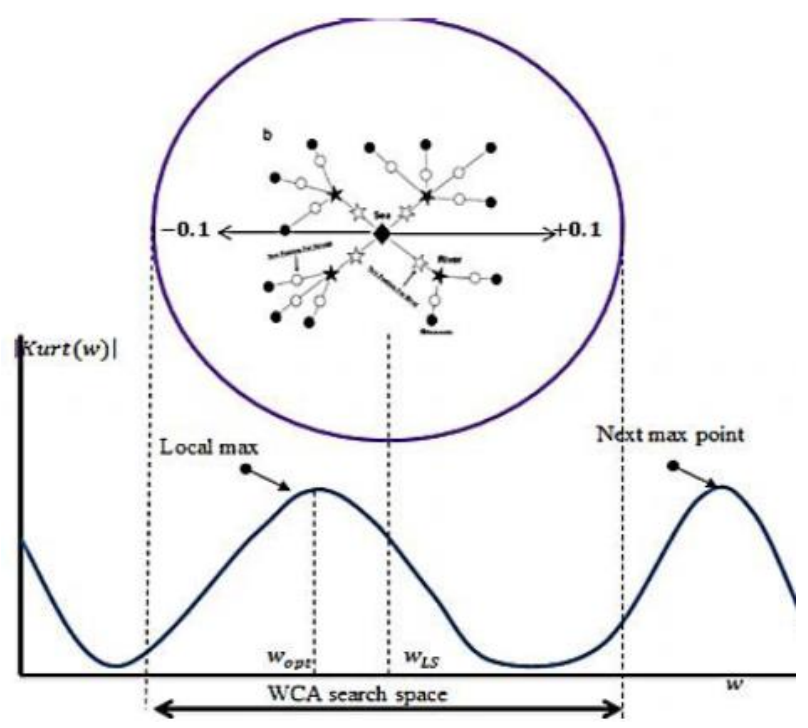

Figure. 6 Initialization for WC algorithm

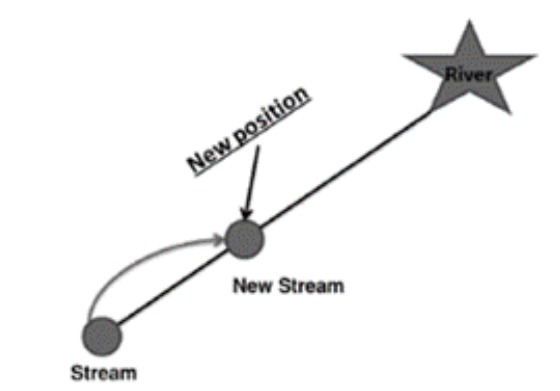

(a)

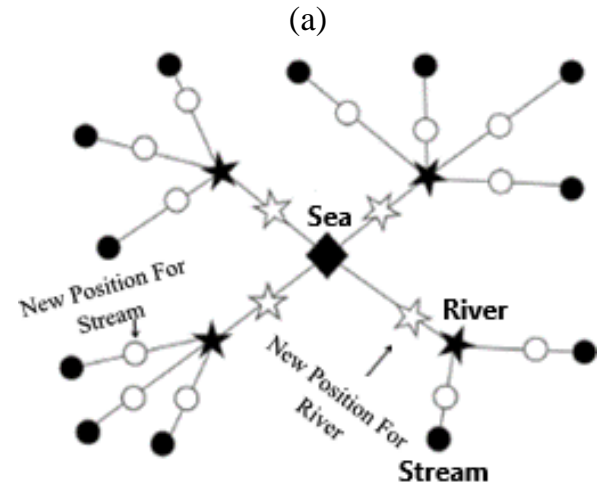

(b)

Figure. 7 The WCA Optimization: (a) Streams flowing into a specific river and (b) WCA Optimization schematically. enough that the exploring space does not reach the maximum points of the neighbor.as shown in Fig. 6.

$N_{p o p}$ are generated in the initial stage. The sea and rivers are then chosen from a group of the best individuals $N_{s r}$ (minimum values). The sea is believed to be the stream with the lowest value (objective function) among the others. In actuality, $N_{s r}$ is the sum of the number of rivers and a single sea (as stated by the user).

A rest of population is viewed as a stream that runs into rivers or directly into the sea. Each river receives water from streams based on the size of the flow. As a result, the quantity of water that enters the river and/or the sea fluctuates from one stream to another. Rivers also flow to the sea, that is the most sloped region.

To assign streams to rivers and the sea based on the intensity of the flow, use the following formula:

$$
\text { Cost }_{i}=f\left(\text { stream }_{i}\right)=f\left(x_{1}, x_{2}, x_{3}, \ldots, x_{4}\right)
$$

where $i=1,2,3, \ldots \ldots, N$

$$
C_{n}=\operatorname{Cost}_{n}-\operatorname{Cost}_{N_{s r}+1}, n=1,2,3, \ldots, N_{s r}
$$

Eq. (30) is used to determine the designated streams for each river and sea.

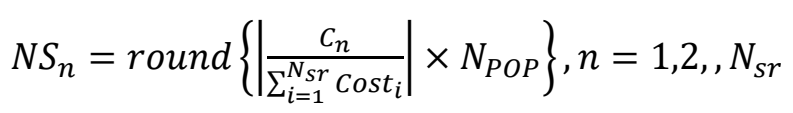

where $N_{s r}$ denotes the streams number that feed into the specified rivers and sea.

Fig. 7 shows the WCA optimization process.

The following equation is used to compute the streams for each river and the sea [20].

$$
N S_{n}=\operatorname{round}\left\{\left|\frac{\operatorname{cost}_{n}-\text { Cost }_{N_{S r}}+1}{\sum_{i=1}^{N} C_{n}}\right| \times N_{\text {Streams }}\right\}
$$

where, $n=1,2, \ldots, N_{s r}$ and the $N_{s r}$ is number of streams that feed into the specified rivers and the sea. Fig. 7(a, b) depicts a schematic representation of a stream moving towards a specific river and the WCA optimization method. For WCA's exploitation phase the following new stream and river sites have been proposed [19]:

$$
\begin{aligned}
& \vec{X}_{\text {Stream }}^{i+1}=\vec{X}_{\text {Stream }}^{i}(t)+\text { rand } \times C \times\left(\vec{X}_{\text {sea }}^{i}(t)-\right. \\
& \left.\vec{X}_{\text {Stream }}^{i}(t)\right)
\end{aligned}
$$




$$
\begin{aligned}
& \vec{X}_{\text {Stream }}^{i+1}=\vec{X}_{\text {Stream }}^{i}(t)+\text { rand } \times C \times\left(\vec{X}_{\text {River }}^{i}(t)-\right. \\
& \left.\vec{X}_{\text {Stream }}^{i}(t)\right) \\
& \vec{X}_{\text {River }}^{i+1}=\vec{X}_{\text {River }}^{i}(t)+\text { rand } \times C \times\left(\vec{X}_{\text {sea }}^{i}(t)-\right. \\
& \left.\vec{X}_{\text {River }}^{i}(t)\right)
\end{aligned}
$$

where $t$ is the iteration index, $1<C<2$ (where 2 is the best value for $\mathrm{C}$ ), where rand is the uniformly distributed random integer between the numbers 0 and 1. Eqs. (32) and (33) are for the streams that run into sea and their comparable rivers. If the solution of stream is better than of its connected river the places of river and stream are swapped (i.e., the stream transforms into a river, and the river transforms into the stream). A river and the sea can be exchanged in a similar way. To avoid early (immature) convergence to local optima, the evaporation process operator is also introduced (exploitation phase Evaporation is the process by which sea water evaporates when rivers/streams flow into it. This causes fresh precipitation. As a result, we must determine if the river/stream is close enough to the sea for evaporation to occur. For this reason, the evaporation condition between a river and the sea is measured using the following criterion:

$$
\text { if }|| \vec{X}_{\text {sea }}^{i}-\vec{X}_{\text {River }}^{i} \|<d_{\max }, i=1,2,3, \ldots, N_{S R}-1
$$

where $d_{\max }$ is a tiny integer around zero. The rainy process is employed after evaporation, and fresh streams emerge in diverse locations (similar to mutation in the GAs). Indeed, the evaporator operator is in charge of the exploratory phase in the WCA. To designate new positions of newly created streams, a uniform random search is utilized. A big $d_{\max }$ number discourages future searches, but a lower value encourages search intensity near the sea. As a result, $d_{\max }$ determines the intensity of the search near the water (i.e., best obtained solution). The value of $d_{\max }$ decreases adaptively as seen below [20]:

$$
d_{\text {max }}^{i+1}=d_{\text {max }}^{i}-\frac{d_{\text {max }}^{i}}{\text { Max Iteration }}
$$

where $t=1,2, \ldots .$. Max_iter

The evolution of the WCA optimization process is depicted in Fig. 7 (b), with circles, stars, and diamonds representing streams, rivers, and the sea, respectively. The white (empty) shapes represent the new locations of streams and rivers.
There are two ways to end the evolution process.

1- The first one: variance of cost function for sea position $\leq$ threshold $=10^{-5}$.

2- The second: if number of iterations $\geq \max$. iteration.

In the evolution step optimum solution for WCA (after iteration stopped) is $\boldsymbol{w}_{\text {opt }}=\boldsymbol{X}_{\boldsymbol{g}}$.

\section{Simulation and results}

In this paper, the QPSK - STBC $(2 \times 1,2 \times 2,2 \times 4$, $4 \times 2$ and $4 \times 4)$ MIMO channel was implemented using the MATLAB-2018 software. The decoding and estimating performances are analysed using the BER and the number of iterations. In this work, a random data generator generates digital information bits (frame by frame), where the frame length changes based on the situation. The QPSK modulator was used to modulate these frames, resulting in the different amount of the symbols for each frame.

The first $N_{p}$ values will be used as the training symbols, with the remaining frame length $N_{t}$ encoded by the STBC encoder serving as the data symbol. Two and four antennas are presented in this paper for transmitting encoded signals over a MIMO STBC Rayleigh fading channel, the received signal is mixed with its complex AWGN. The training symbols are used to compute the channel estimate coefficient in the LS channel estimation. At the receiver end, the MRC is being used to decode the other received symbols, which are then transmitted to the QPSK demodulator. In order to calculate a BER for the given SNR, the coded bits are compared to the original data bit frame. Second, simple criteria based on the use of $H_{L S}$ (calculated channel coefficient using a LS estimator with restricted experimental sequence of training) is used as the beginning value of the statistically based blind channel estimator to produce a semi blind channel estimator. The performance of channel estimation for MIMO STBC was described using nine parameters: number of transmitter antennas, number of receiver antennas, SNR, BER, the number of iterations, numeral of frame, number of symbols/frames, number for pilot, and sampling rate as follows $[15,16]$.

\subsection{BER performance of ICA algorithms}

This section compares the BER performance for channel estimation when the kurtosis type of ICA method is used for one source to the BER performance for channel estimation when the kurtosis is coupled with the WCA as follows: 


$$
\text { A- } 2 \times 1, N_{p}=8 \text { : }
$$

Using kurtosis-based ICA with number of samples/frames $=1024$, the simulation result is shown in Fig. 8 (a, b)

\section{B- $2 \times 2, N_{p}=8$ :}

By using kurtosis-based ICA and number of samples/frames $=1024$, the simulation result is shown in Fig. 9 (a, b)

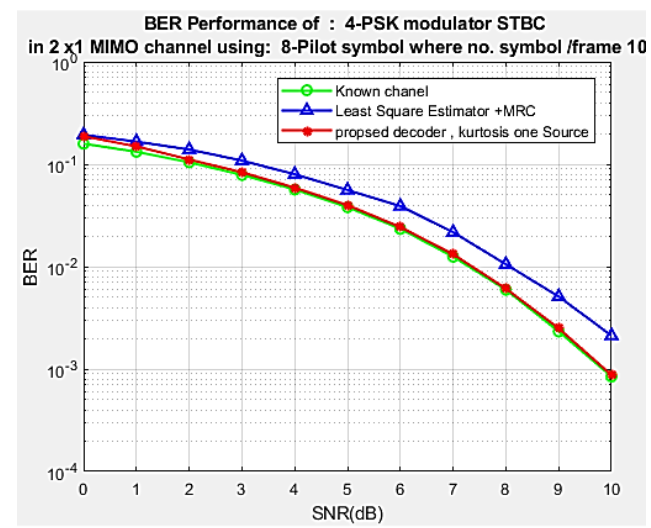

(a)

\section{C- $4 \times 2, N_{p}=8$ :}

By using kurtosis-based ICA and number of samples/frames $=1024$, the simulation result is shown in Fig. $10(a, b)$

\section{D- $4 \times 4, N_{p}=8$ :}

By using kurtosis-based ICA and number of samples/frames $=1024$, the simulation result is shown in Fig. 11 (a, b).

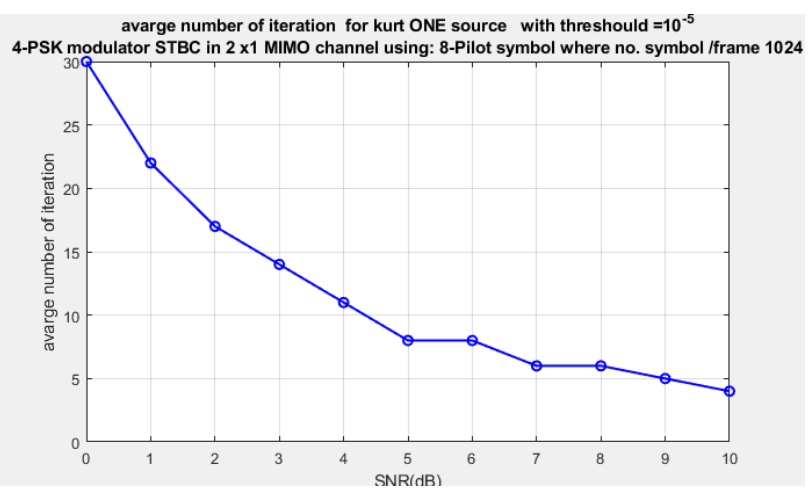

(b)

Figure. 8 (a) BER performance of one source kurtosis based ICA (b) average number of iterations for kurtosis one source

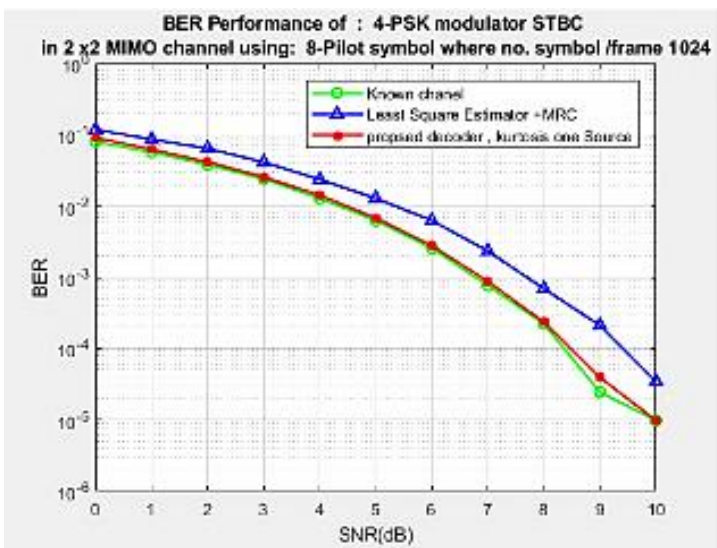

(a)

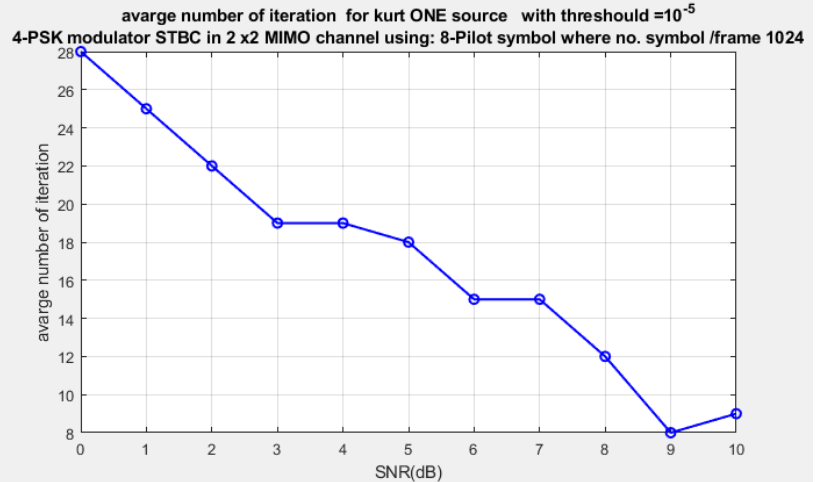

(b)

Figure. 9 (a) BER performance of one source kurtosis based ICA (b) average number of iterations for kurtosis one source

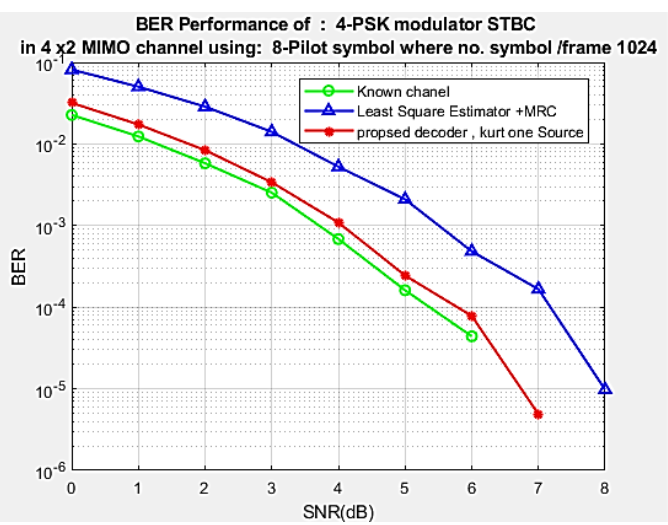

(a)

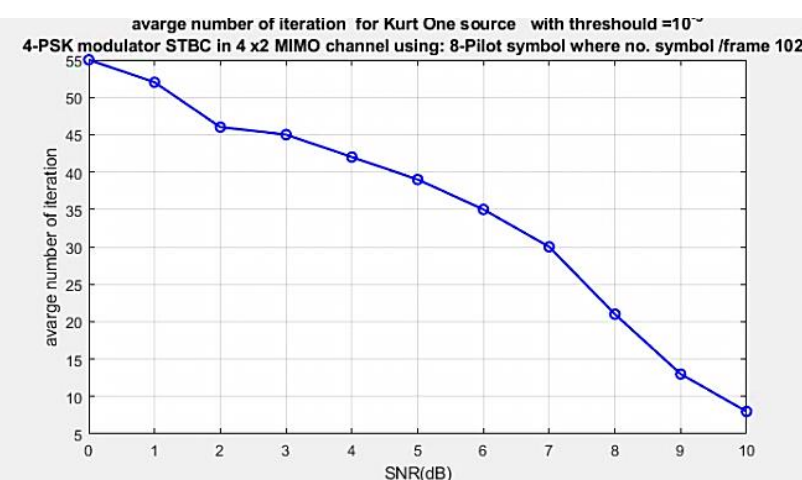

(b)

Figure. 10 (a) BER performance of one source kurtosis based ICA (b) average number of iterations for kurtosis one source 


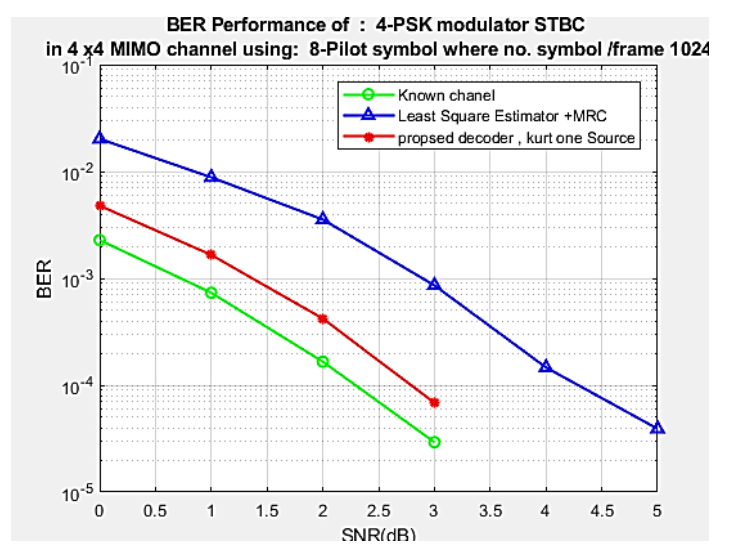

(a)

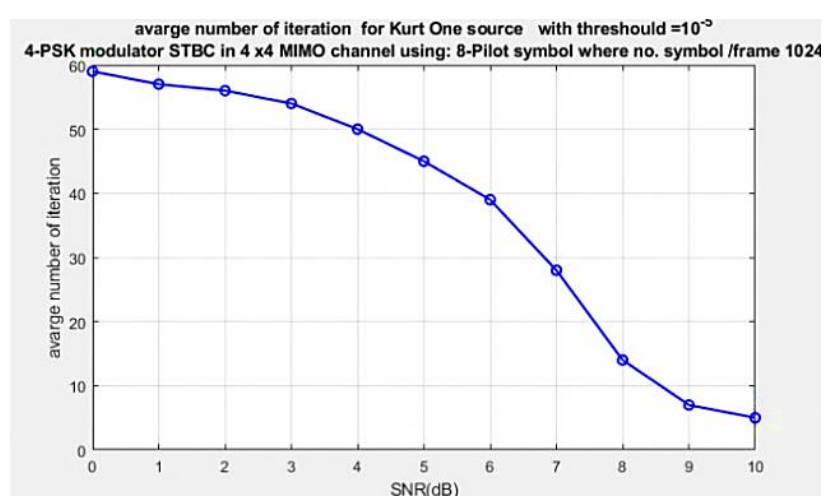

(b)

Figure. 11 (a) BER performance of one source kurtosis based ICA and (b) average number of iterations for kurtosis one source

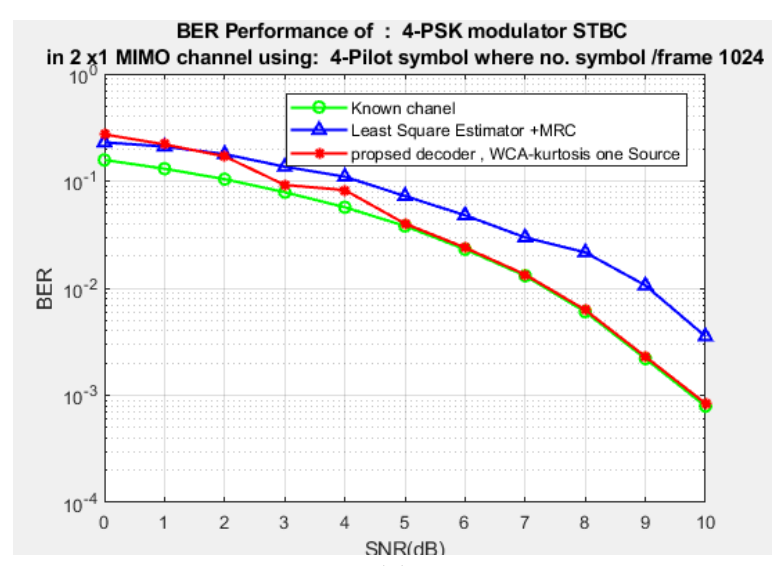

(a)

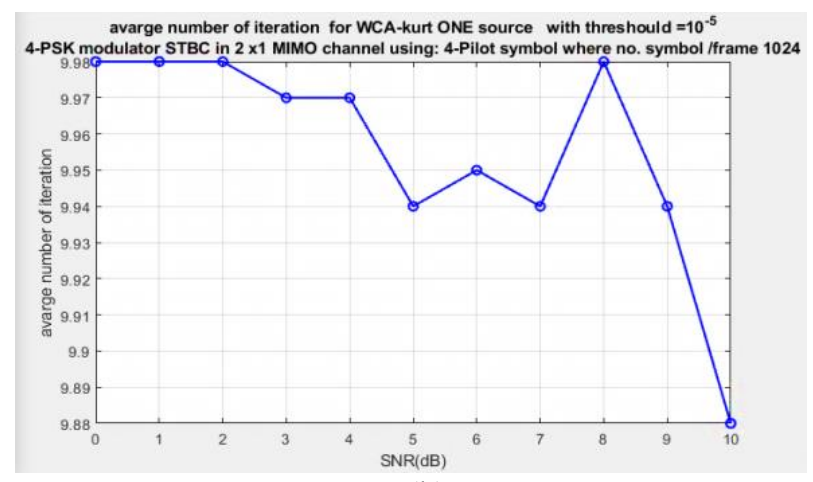

(b)

Figure. 12 (a) BER performance of one source WCA-kurtosis based ICA and (b) average number of iterations for WCA-kurtosis one source.

\subsection{Comparison between BER performance of WCA-kurtosis and BER performance kurtosis}

In this section, the BER performance of WCAkurtosis and kurtosis-ICA had been compared in terms of BER performance and average number of iterations with in SNR varied numbers of frames.

\section{A- $2 \times 1, N_{p}=8$ :}

By using WCA-kurtosis based ICA and number of samples/frames $=1024$, the simulation result is shown in Fig. 12 (a, b).

From the calculation of BER optimization at $10^{-2}$ for the two antennas at the transmitter, we show that the performance of the proposed algorithm using WCA-kurtosis is better than the performance of the LS algorithm by $1.6 \mathrm{~dB}$ at the length of the 1024 frame which is shown in Fig. 12 (a). The performance of the proposed algorithm using kurtosis-ICA shows $0.6 \mathrm{~dB}$ better than the LS algorithm in the 1024 frame length shown in Fig. 8 (a).

\section{B- $2 \times 2, N_{p}=8$ :}

By WCA-kurtosis based ICA and number of samples/frames $=1024$, the simulation result is shown in Fig. 13 (a, b).

From the calculation of BER optimization at $10^{-4}$ for the two antennas at the transmitter, we show that the performance of the proposed algorithm using WCA-kurtosis is better than the performance of the LS algorithm by $1.5 \mathrm{~dB}$ at the length of the 1024 frame which is shown in Fig. 13 (a). The performance of the proposed algorithm using kurtosis-ICA shows $1 \mathrm{db}$ better than the LS algorithm in the 1024 frame length shown in Fig. 9 (a).

\section{C- $4 \times 2, N_{P}=8$ :}

By using WCA-kurtosis based ICA and number of symbole/frame $=1024$, the simulation result is shown in Fig. 14 (a, b).

From the calculation of BER optimization at $10^{-4}$ for the tow antenna at the transmitter, we show 
that the performance of the proposed algorithm using WCA-kurtosis is better than the performance of the LS algorithm by $2.1 \mathrm{db}$ at the length of the 1024 frame which is shown in Fig. 14 (a) The performance of the proposed algorithm using kurtosis-ICA shows $1.2 \mathrm{~dB}$ better than the LS algorithm in the 1024 frame length shown in Fig. 10 (a).

\section{D- $4 \times 4, N_{P}=8$ :}

By using WCA-kurtosis based ICA and number of symbole/frame $=1024$, the simulation result is shown in Fig. 15 (a) and (b).

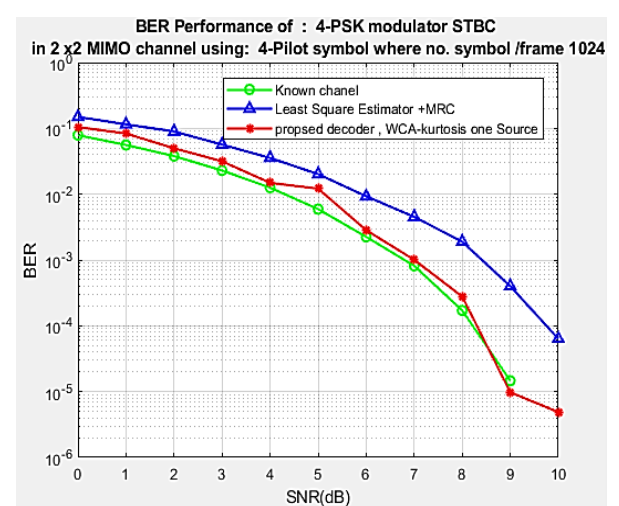

(a)

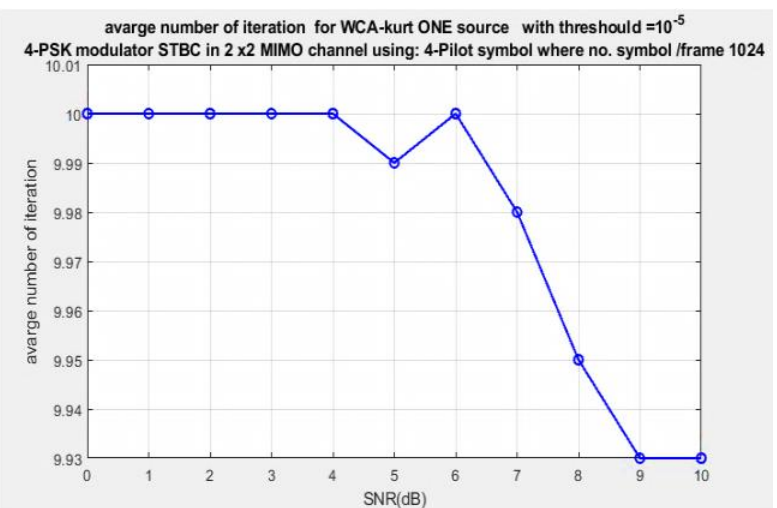

(b)

Figure. 13 (a)BER performance of one Source WCA-kurtosis based ICA and (b) average number of iterations for WCAkurtosis one source

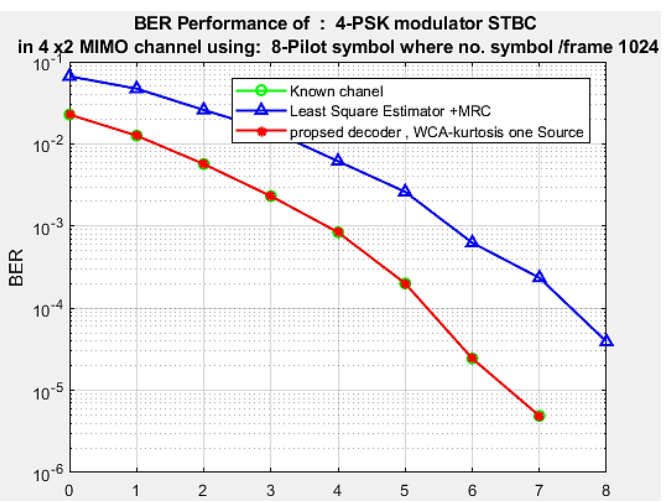

(a)

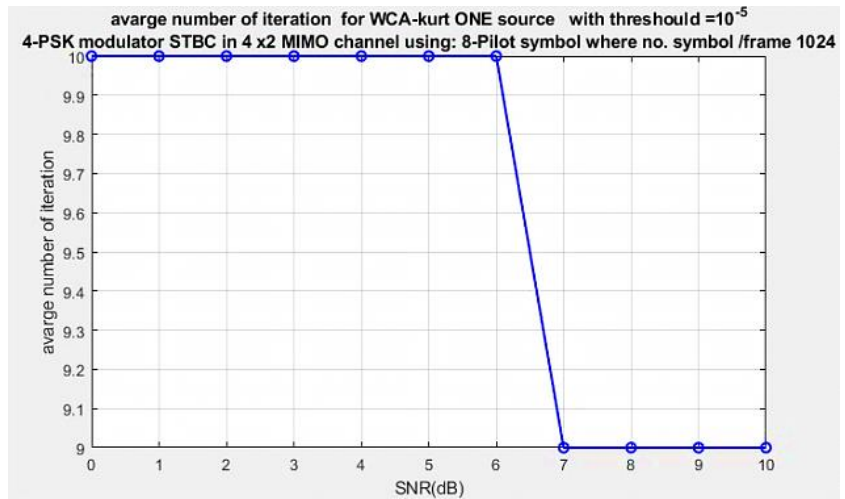

(b)

Figure. 14 (a) BER performance of one source WCA-kurtosis based ICA and (b) average number of iterations for WCAkurtosis one source
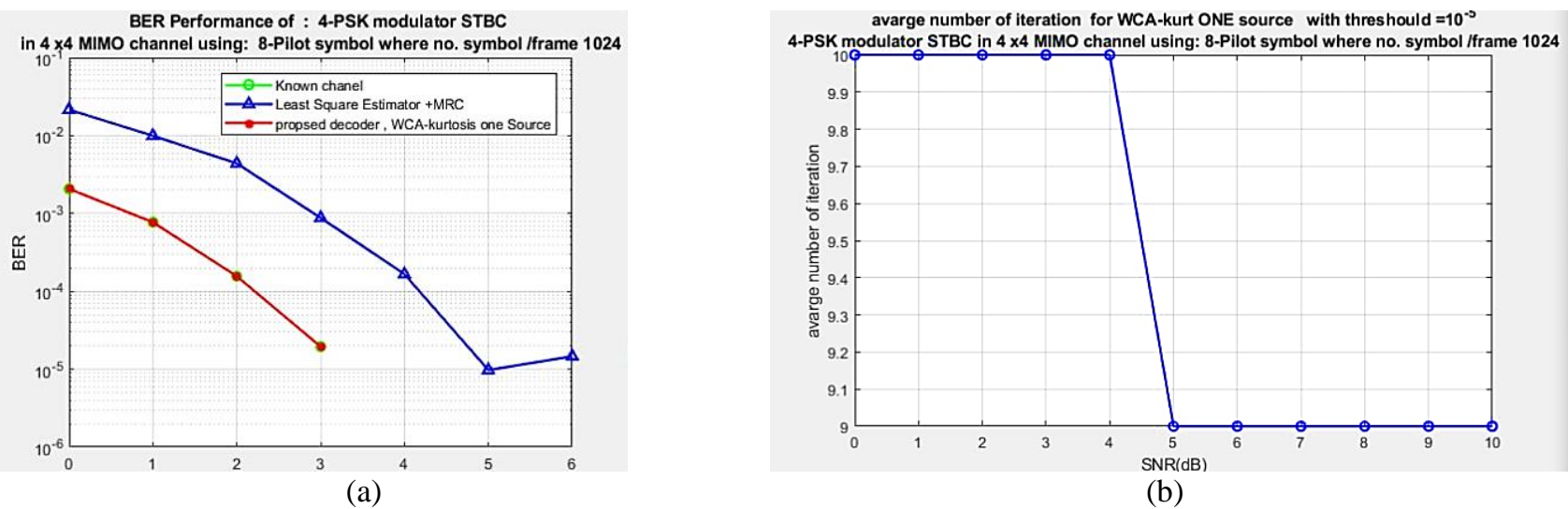

(b)

Figure. 15 (a) BER performance of one source WCA-kurtosis based ICA and (b) average number of iterations for WCA-kurtosis one source. 
From the calculation of BER optimization at $10^{-4}$ for the two antennas at the transmitter, we show that the performance of the proposed algorithm using WCA-kurtosis is better than the performance of the LS algorithm by $2.2 \mathrm{~dB}$ at the length of the 1024 frame which is shown in Fig. 15 (a) The performance of the proposed algorithm using kurtosis-ICA shows $2.8 \mathrm{~dB}$ better than the LS algorithm in the 1024 frame length shown in Fig. 11(a).

\section{Conclusion}

This study introduces a fresh method for the direct decoding the MIMO STBC system based on modelling MRC as a mixing function. This method eliminates the requirement for the separate estimator. Despite fact that the modern suggested decoder is considerably more complex to implement than existing decoders, the new decoder, according to MRC, is better. By using fewer pilot symbols, BER performance can be improved. An MRC model is used to decode this data. For BSS techniques, the matrix of channel mixes is used instead of a mixed matrix. Despite the fact that a MIMO channel has fewer receive antennas than a broadcast channel, the new model allows for the usage of BSS. The most important element of the method is that the entire system is decoded using only one source. This criterion reduces decoding time to $1 / n_{s}$ of a second. The problem of source and sign ambiguity in BSS solved by use a proper initialization of a de mixing vector.

The standard WC algorithm is modified in this study by developing a new update formula that incorporates population behaviour and the GAA algorithm. It has also been discovered that integrating WCA with the kurtosis BSS based on one source extraction offers a high speed, low complexity, and BER 0ptimized solution. As a consequence, any MIMO-STBC system will operate with the proposed decoder.

\section{Conflicts of interest}

The authors declare no conflict of interest.

\section{Author contributions}

The paper methodology, software, validation, formal analysis; investigation, resources, data curation, writing - original draft preparation, and funding acquisition have been done from the first author.

Conceptualization, writing - review and editing, visualization, supervision, and project administration have been done from the second author.

\section{References}

[1] S. M. Alamouti, "A Simple Transmit Diversity Technique for Wireless Communications", IEEE Journal on Select Areas in Communications, Vol. 16, No. 8, pp. 1451-1458, 1998.

[2] S. G. Glisic, Advance Wireless Communications, $2^{\text {nd }}$ Edition Johan Wiley \& Sons, Ltd, 2007.

[3] J. R. Hampton, Introduction to MIMO Communications, Cambridge University Press, 2014.

[4] G. Qian, L. Li, and M. Luo, "On the Blind Channel Identifiability of MIMO-STBC Systems Using Noncircular Complex FastICA Algorithm", Circuits, Systems, and Signal Processing, Vol. 33, No. 6, pp 1859-1881, 2014.

[5] V. Tarokh, H. Jafarkhani, and A. R. Calderbank, "Space-Time Block Codes from Orthogonal Design", IEEE Transactions on Information theory, Vol. 45, No. 5, pp. 1456-1467, 1999.

[6] V. Tarokh, H. Jafarkhani, and A. R. Calderbank, "Space-Time Block Coding for Wireless Communications: Performance Results", IEEE Journal on Selected Areas in Communications, Vol. 17, No. 3, pp. 451-460, March 1999.

[7] L. A. Perişoară, "BER Analysis of STBC Codes for MIMO Rayleigh Flat Fading Channels", Telfor Journal, Vol. 4, No. 2, pp. 78-82, 2012.

[8] S. D. Santumon and B.R. Sujatha, "Space-Time Block Coding (STBC) for Wireless Networks", International Journal of Distributed and Parallel Systems (IJDPS), Vol. 3, No. 4, pp. 183-195, July 2012.

[9] M. Biguesh and A. B. Gershman, "TrainingBased MIMO channel Estimation: A Study of Estimator Tradeoffs and Optimal Training Signals", IEEE transactions on signal processing, Vol. 54, No. 3, pp.884-893, 2006.

[10] C. Budianu and L. Tong, "Channel Estimation for Space-Time Orthogonal Block Codes", IEEE Transactions on Signal Processing, Vol. 50, No. 10, pp. 2515-2528, 2002.

[11] G. Taricco and E. Biglieri, "Space-Time Decoding with Imperfect Channel Estimation", IEEE Transactions on Wireless Communications, Vol. 4, No. 4, pp. 1874-1888, 2005.

[12] A. Hyvärinen and E. Oja, "Independent Component Analysis: Algorithms and Applications", Neural networks, Vol. 13, No. 4, pp. 1-31, 2000.

[13] A. Hyvarinen, "Fast and Robust Fixed-Point Algorithms for Independent Component 
Analysis", IEEE Transactions on Neural Networks, Vol. 10, No. 3, pp. 626-634, 1999.

[14] Z. Koldovsky, P. Tichavsky, and E. Oja, "Efficient Variant of Algorithm FastICA for Independent Component Analysis Attaining the Camer - Rao Lower Bound", IEEE Transactions on Neural Networks, Vol. 17, No. 5, pp. 12651277, 2006.

[15] K. K. Abdalla and S. A. K. Alrufaiaat, "A New Robust Decoding Technique of Four Transmitters MIMO STBC System based on FastICA Algorithm", International Journal of Intelligent Engineering and System, Vol. 14, No. 1, pp. 181-191, 2021.

[16] N. S. Ali, K. K. Abdalla, and S. A. Kadhim, "BER Performance Improvement of Alamouti MIMO-STBC Decoder Using Mutual Information Method", Journal of Physics: Conference Series, Vol. 1530, No. 1, 2020.

[17] T. H. Liu, "Comparisons of Two Real-Valued MIMO Signal Models and Their Associated ZFSIC Detectors over the Rayleigh Fading Channel", IEEE Transactions on Wireless Communications, Vol. 12, No. 12, pp. 60546066 December 2013.

[18] L. A. Perişoară, "BER analysis of STBC codes for MIMO Rayleigh flat fading channels", Telfor Journal, Vol. 4, No. 2, pp. 78-82, 2012.

[19] H. Eskandar, A. Sadollah, and A. Bahreininejad, "Water cycle algorithm-A novel metaheuristic optimization method for solving constrained engineering optimization problems", Computers \& Structures, Vol. 110 pp. 151-166, 2012.

[20] A. Sadollah, H. Eskandar, and A. Bahreininejad, "Water cycle algorithm with evaporation rate for solving constrained and unconstrained optimization problems", Applied Soft Computing, Vol. 30, pp. 58-71, 2015.

[21] S. P. Jadhav and V. S. Hendre, "Performance of maximum ratio combining (MRC)MIMO systems for Rayleigh fading channel", International Journal of Scientific and Research Publications, Vol. 3, No. 2 pp. 1-4, 2013.

[22] L. A. Perişoară, "BER analysis of STBC codes for MIMO Rayleigh flat fading channels" Telfor Journal, Vol. 4, No. 2, pp. 78-82, 2012.

[23] J. Vía, I. Santamaría, and J. Pérez, "Code combination for blind channel estimation in general MIMO-STBC systems", Journal on Advances in Signal Processing, pp. 1-12, 2009. 